Article

\title{
Structural Characterization of Graphite Nanoplatelets Synthesized from Graphite Flakes
}

\author{
Hani Manssor Albetran 1,* \\ 1 Department of Basic Sciences, College of Education, Imam Abdulrahman Bin Faisal University, P. O. Box \\ 2375, Dammam 31451, Saudi Arabia; halbatran@iau.edu.sa (H.M.A.) \\ * Correspondence: halbatran@iau.edu.sa (H.M.A.); Tel.: +966 13827 4155; Fax: +966 138269936.
}

Received: date; Accepted: date; Published: date

\begin{abstract}
Graphite nanoplatelets (GNPs) were produced from flake graphite that had been immersed in isopropyl alcohol solution (70\%) and converted to powder form in an ultrasonic bath ( $5 \mathrm{~h}$, room temperature). Scanning and transmission electron microscopy, $\mathrm{X}$-ray diffractometry, and Raman spectroscopy identified 120-nm-thick GNP crystallites and 0.5-21 $\mu \mathrm{m}$ plates with different areas and shapes. Extensive exfoliation was observed by transmission electron microscopy with abundant multilayer and some monolayer GNPs. X-ray diffractometry confirmed 43 GNP layers along the c-axis. Raman spectroscopy indicated well-defined GNPs with few defects and no oxide content. Rietveld analysis indicated a GNP crystal lattice with stacks of parallel two-dimensional graphene layers and tightly bound hybridized carbon atoms stacked in a translational ...ABAB... sequence in hexagonal rings.
\end{abstract}

Keywords: Carbon; Crystal structure; Graphene; Graphite; Nanoplatelet.

\section{Introduction}

As the sixth element in the periodic table, carbon can catenate with elemental carbon or other elements to form organic structures as the basis of life. Zero-, one-, two-, and three-dimensionalstructures, which are termed fullerenes, carbon nanotubes, graphene, and graphite, respectively, are basic elemental carbon-based structures [1,2].

Graphite, as one of the oldest known forms of carbon and termed black lead or plumbago, which in Greek "grapho", means "to write" and is widely used in pencils and lubricants. Graphite occurs in metamorphic rocks (sedimentary carbon-compound reduction during metamorphism), in igneous rocks, and in meteorites [3]. Graphite is used in electronic products, such as batteries, electrodes, and solar panels, because of its high conductivity, and low cost. In addition, its high thermal conductivity, and low mass make it an ideal thermal conductive polymer composite in manufacturing [3-7].

Despite graphite appearing to be a well-defined homogeneous product with a known chemical and physical structure, different properties are displayed by different graphite types (amorphous, flake, vein, and synthetic). The two forms or phases of graphite include rhomobohedral and hexagonal crystal structures with similar physical properties (density $=1.9 \mathrm{~g} / \mathrm{cm}^{3}$, iron-black to steelgray color, and deep blue in transmitted light). The hexagonal and rhombohedral graphite phases have a $\mathrm{P}_{63} / \mathrm{mcc}$ and $\mathrm{R}_{3} \mathrm{~m}$ space group, 4 and 6 atoms per unit cell $(Z)$, and $\mathrm{a}=0.2461 \mathrm{~nm}$ and $\mathrm{c}=0.6708$ $\mathrm{nm}$ and $a=2.456 \mathrm{~nm}$ and $c=10.044 \mathrm{~nm}$ lattice parameters at room temperature, respectively [8,9]. The single-crystal lattice stacking periodicity structure of the hexagonal $(2 \mathrm{H})$ and rhombohedral $(3 \mathrm{R})$ graphite phases is $A B A B$ and $A B C A B C$, respectively. Graphite can be cleaved along basal planes and its particle size is readily reduced by mechanical grinding because the interlayer bonding force is weaker than that of the intralayers [10-16]. Graphene, which is the term for individual graphite layers, can be restacked to prepare graphite [17]. In the stacking, carbon atoms are layered in 0.142$\mathrm{nm}$-spaced honeycomb lattices, and planes are separated by $0.335 \mathrm{~nm}$ [5]. Their similar structures prevent characterization techniques from being used to distinguish between graphite and graphene [18]. 
Graphite nanomaterials are of interest because of their safe, affordable, stable, nontoxic, noncarcinogenic, bactericidal, photocatalytic, industrial, biomedical, and wastewater treatment applications [7,19]. Carbon nanostructures can be produced from graphite, such as three-dimensional carbon nanotubes and vapor-grown carbon nanofibers from the coiling of covalent graphite building [20], diamond-like sp3 and fullerene-like bonding from ball milling for up to $1000 \mathrm{~h}$ in vacuum [15] (structural changes are of scientific importance with wide potential for application [11-16], and graphite nanoplatelet graphitic nanofillers (GNPs, also termed graphite nanosheets, graphite nanoflakes or exfoliated or expanded graphite) from randomly stacked two-dimensional graphene sheets [4].

In this study, scanning and transmission electron microscopy (SEM and TEM, respectively), Xray diffractometry (XRD, with Rietveld refinement), and Raman spectroscopy were used to determine the physicochemical properties of GNPs that were synthesized from flake graphite.

\section{Experimental methodology}

\subsection{Material synthesis}

Graphite flakes (molecular weight $12.01 \mathrm{~g} / \mathrm{mol}$, product number 332461, Sigma-Aldrich) were used to prepare the GNPs by immersion in isopropyl alcohol $(70 \%)$ and ultrasonication with frequency of $40 \mathrm{kHz}$ in a bath for $5 \mathrm{~h}$ at room temperature, which resulted in shear forces and cavitation by micron-sized bubble growth and collapse. GNPs were formed by filtering and drying the product dispersion.

\subsection{Materials characterization}

\subsubsection{SEM}

The GNP morphology was studied by SEM (IRMC-INSPECT S50, $20 \mathrm{kV}$ acceleration voltage). The GNPs were not coated to prevent charging because they are a good electrical conductor.

\subsubsection{TEM}

Ground samples of GNPs ( $\sim 5 \mathrm{mg}$ ) were ultrasonicated in an ethanol suspension for $10 \mathrm{~min}$. TEM imaging (FEI MorgagniTM 268, tungsten tip electron source, $100 \mathrm{kV}$ ) was carried out on copper grids with two drops of suspension.

\subsubsection{Raman spectroscopy}

Raman spectroscopy was undertaken on a Labram 1B dispersive Raman spectrometer (2 mW, 150$\mu \mathrm{m}$ slit, $632.817 \mathrm{~nm}$ excitation, 50× objective, 600 lines $/ \mathrm{mm}$ diffraction grating, Peltier-cooled chargecoupled device detector at $-40{ }^{\circ} \mathrm{C}$, with $60 \mathrm{~s}$ collection time and $10 \mathrm{~s}$ for silicon). The silicon peak correction was undertaken by shifting the Raman spectra by a 520.7 wavenumber.

\subsubsection{XRD}

The GNP purity and structure were confirmed by XRD (Rigaku Benchtop Miniflex X-ray diffractometer, $\mathrm{Cu}-\mathrm{K}_{\alpha}$ radiation, $\lambda=0.1541 \mathrm{~nm}, 40 \mathrm{kV}, 20 \mathrm{~mA}, 20-70^{\circ}, 0.02 / \mathrm{min}$ scanning speed). FullProf software (version 7.20) and Rietveld pattern analysis was used with the goodness-of-fit obtained from the weighted pattern R-factor $\left(R_{w p}\right)$, derived Bragg R-factors ( $\left.R \mathrm{P}\right)$, and expected R-factor (Rexp). An optimized sample displacement, pattern background, peak shape, preferred orientation, $2 \theta_{0}$, scale factor, and lattice parameters were used to calculate the GNP crystalline phase abundances. Rietveld refinements were undertaken by using the Crystallography Open Database and graphite crystal structures (COD 9000046). 


\section{Results and discussion}

\subsection{GNP microstructures}

SEM and TEM were used to determine the particle size, morphology, and microstructure of GNPs. Typical secondary electron images of sonicated graphite flakes are presented in Figure 1a and (b). Fragmented flake graphite yielded foliated parallelogram graphite plates. The ImageJ ${ }^{\circledR}$ software (National Institutes of Health, USA, version 1.48e) was used to determine the area and thickness of 70 GNPs, and they were $0.5-21 \mu \mathrm{m}$ and $\sim 120 \mathrm{~nm}$, respectively. These values are comparable with the nanosheets that Chen et al. obtained by ultrasonic of graphite powder [21].
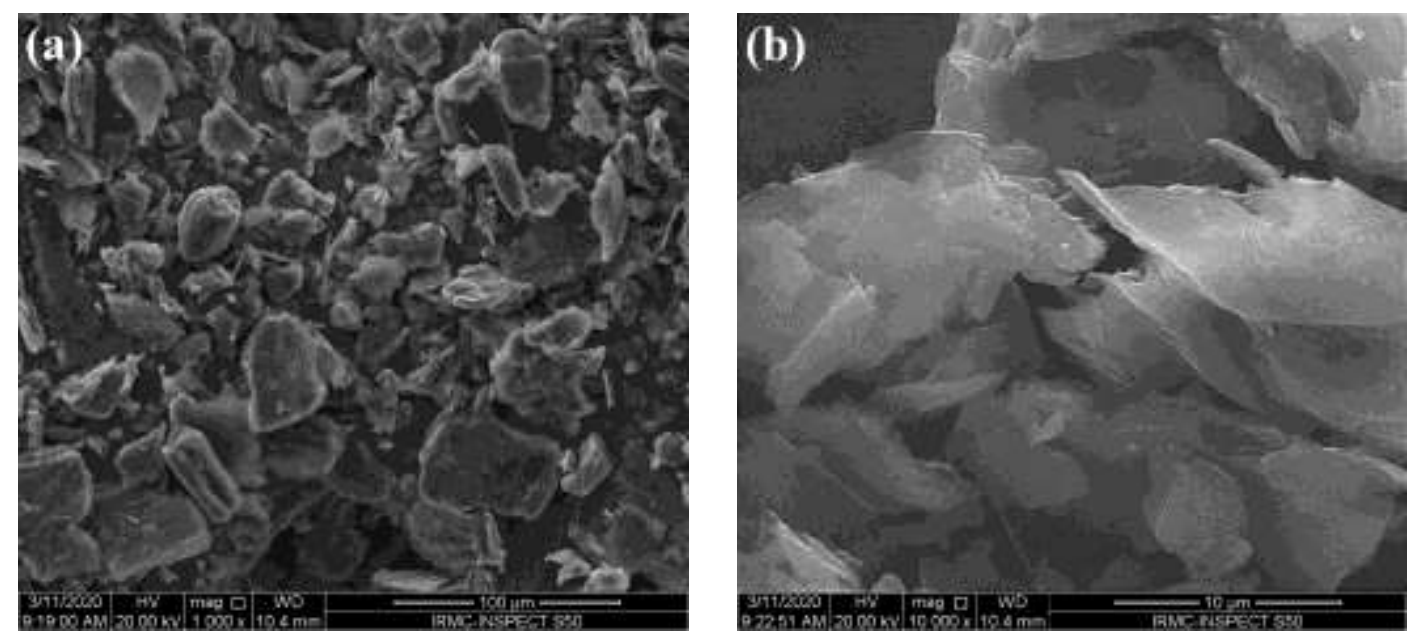

Figure 1. (a) Low- and (b) high-resolution SEM micrographs of GNPs.

The TEM micrographs in Figure 2a and b show graphite in nanoplatelets at a low resolution and granularity in the higher-resolution images, respectively. Therefore, nanoplatelets with varying shapes and areas formed because ultrasonication prior to TEM broke down graphite nanoplatelets into smaller pieces. The weak interlayer van der Waals attraction allowed the nanoplatelets to slide past each other perpendicular to the c-axis, but the sufficiently strong attraction prevented the complete formation of individual graphene layers [17]. TEM diffraction contrast from thickness variations indicates that graphite nanoplatelets of a few layers, including bi- and tri-layers, formed, but some monolayer graphene was also visible. Polycrystalline graphite with randomly oriented crystallites with an interplanar spacing of $\sim 0.40 \mathrm{~nm}$ is shown in Figure 2c. Single crystals in natural flake graphite were oriented in a preferred direction, whereas synthetic graphite was oriented more randomly [22].
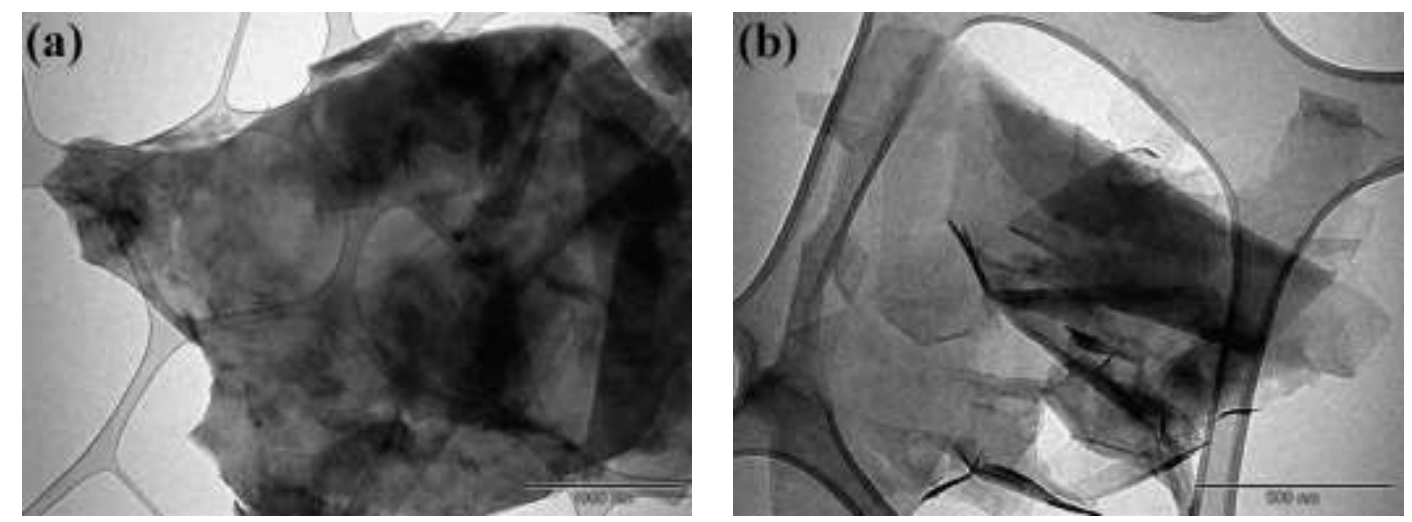


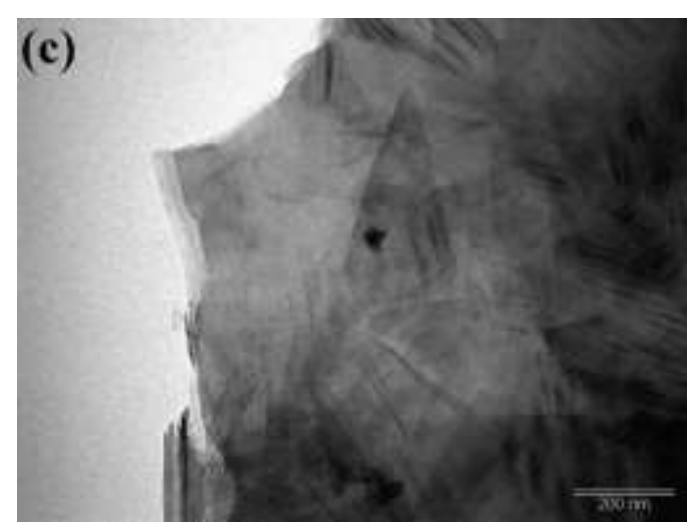

Figure 2. (a) Low-resolution and (b) \& (c) lattice-resolved HR-TEM micrographs of GNPs with polycrystalline graphite material and $\sim 0.33627 \mathrm{~nm}$ d-spacing between adjacent (002) lattice planes.

\subsection{Raman spectroscopy}

Raman spectra of GNPs are shown in Figure 3, where the G and two-dimensional (2D) band shape, position and intensity can be used to estimate the number of GNP layers. The 2D band changes position, width, and shape as the number of layers increases but the peak position of the $G$ band shifts downwards as the layer number increases. Characteristic GNP peaks exist in the D, 2D, and G bands at 1,331, 2,686, and 1,577 $\mathrm{cm}^{-1}$, respectively. A high sample quality is indicated by the weak and strong D and $\mathrm{G}$ peaks (for $\mathrm{sp}^{2}$ carbon), respectively, and the broad multi-band 2D peak indicates multi-layer graphite features [17].

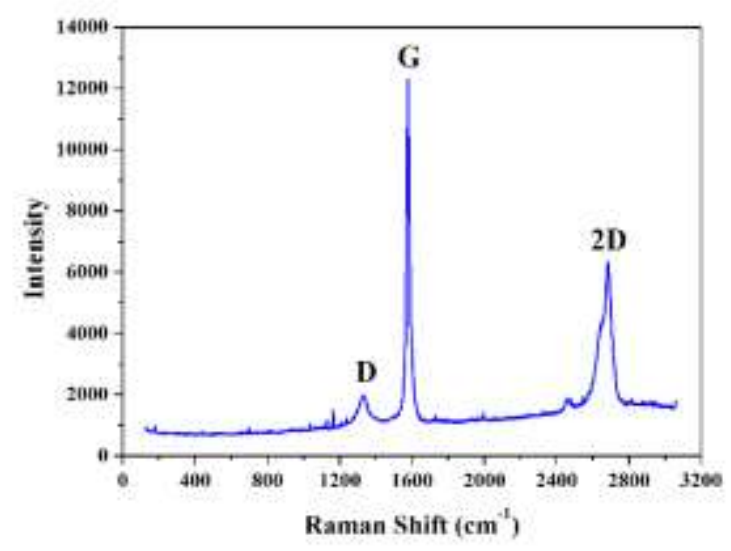

Figure 3. Raman spectra of GNPs.

A primary graphene band characteristic is illustrated by the $\mathrm{sp}^{2}$ carbon atom vibration in the $\mathrm{G}$ band. The D band provides a disordered GNP vibrational peak and is used to characterize GNP structural defects [23]. Well-defined GNPs with few defects are present. Raman-active defect concentrations in parts per million (ppm) are calculated from the ratio of the defect density (nD) and number of carbon atoms (nc) [24,25]:

$$
\begin{aligned}
& \frac{n_{D}}{n_{C}}=\frac{2.16 \times 10^{11}}{3.82 \times 10^{15}} \frac{I(D)}{I(G)} \\
& n_{c}=3.82 \times 10^{15} \mathrm{~cm}^{-2}
\end{aligned}
$$

where $I(D)$ and $I(G)$ are the D- and G-band integration area, respectively, and the concentration defects and integral area ratio are $\sim 0.18$ and $\sim 11 \mathrm{ppm}$, respectively. The measured $I(D) / I(G)$ ratio and 
GNP defect concentrations compared well with the literature values for samples of graphite and graphene [25].

\section{3. $X R D$}

Figure 4 shows the diffraction pattern of crystalline GNP structures. The hexagonal crystal structure of GNP with no impurity or second-phase peaks is shown by characteristic diffraction peaks that were indexed as (002), (020), (111), and (004) planes [11,25].

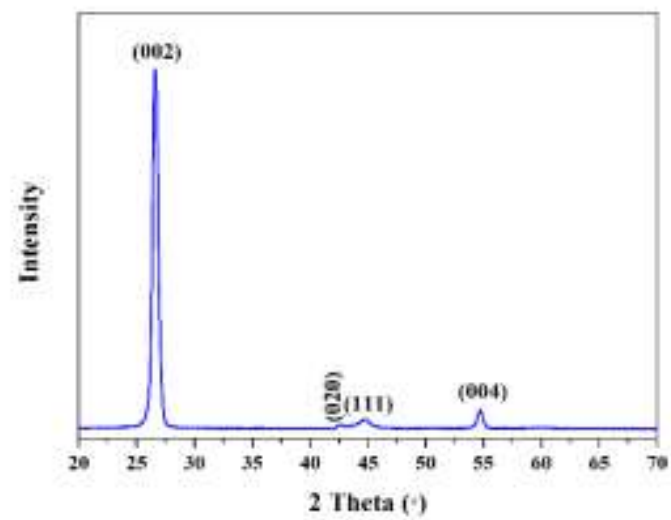

Figure 4. XRD pattern of GNPs.

The reflection profile broadening of XRD pattern is used to calculate the crystallite size and the corresponding peak position is used to determine the interlayer spacing. The average crystallite size $\left(L_{c}\right)$ (c-axis crystalline dimension) of GNP was determined from the Scherrer equation and the do02 (dspacing for $2 \mathrm{H}(002)$ from $2 \theta$ peak at $26.619^{\circ}$ ) was calculated from the Bragg's Law $[25,26]$ :

$$
\begin{aligned}
& L_{c}=\frac{k \lambda}{\beta \cos \theta} \\
& d_{002}=\frac{n \lambda}{2 \sin \theta}
\end{aligned}
$$

where $\theta, \beta, \lambda$, and $k$ are the Bragg angle, line broadening at half maximum intensity (full width at half maximum, radians), X-ray wavelength $(0.15419 \mathrm{~nm})$, and shape factor $(0.91)$, respectively. The computed crystallite size of $14.42 \mathrm{~nm}$ compared well with the graphite sample sizes obtained by Gen et al. [21]. The physical origins of the broad (002)-like reflections are interpreted from the uniform interlayer spacing (d002) and are related directly to layer misalignment with average interlayer spacings like crystalline graphite $(0.335 \mathrm{~nm})$, and these results agree with the TEM micrographs. Pure crystalline graphite has an identical basal spacing [25,27]. Seehra et al. described the Nc number of layers along the c-axis as [24]:

$$
N_{c}=\frac{L_{c}}{d_{002}}
$$

They calculated the interlayer spacing $\left(d_{002}\right)$ and apparent crystallite size $\left(L_{c}\right)$ in the c-direction that provide the numbers of GNP layers. Forty-three GNP layers exist along the c-axis.

\subsection{XRD Rietveld analysis}

Rietveld pattern-fitting of the XRD pattern was analyzed from the FullProf software and the goodness-of-fit was estimated from the derived Bragg R-factors $\left(R_{B}\right)$, expected R-factor $\left(R_{e x p}\right)$, and weighted pattern R-factor $(R w p)$ [28-33]. The preferred orientation, peak shape parameters, lattice parameters, scale factor, $2 \theta_{0}$, sample displacement, and pattern background were optimized in the 
Rietveld refinement to calculate the GNP lattice parameters. The residual GNP XRD Rietveld plots are shown in Figure 5 where the refinements were obtained from graphite crystal structures (COD 9000046). The solid black line and cycles indicate the calculated and measured pattern, respectively, with the difference between the two indicated by a blue residual plot. Green bars show the (002), (020), and (004) peak positions. The residual Rietveld refinements were 5.62 and $\sim 11.9$ for the $R_{w p}$ and Rexp, respectively. The goodness-of-fit $\left(\chi^{2}\right)$ of 4.46 shows an acceptable refinement quality [28-33].

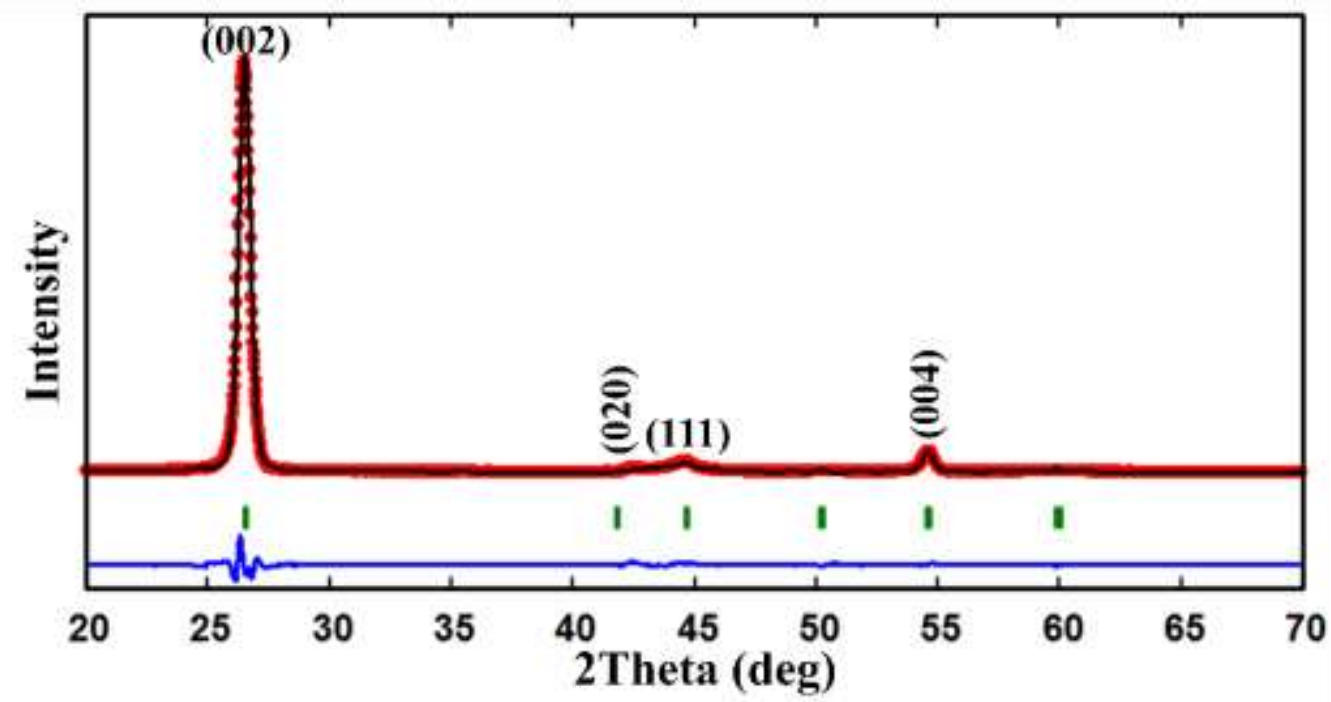

Figure 5. GNPs XRD Rietveld difference plots. Black cycles and solid black line are measured and calculated pattern, respectively. Blue residual plot and green bars indicate the difference between the calculated and measured pattern and peak positions ((002), (020), and (004)), respectively.

A comparison of the density, cell volume, and cell parameters of GNP with literature data is provided in Table 1 . The calculated density of $2.28 \mathrm{~g} / \mathrm{cm}^{3}$, cell volume of $0.06996 \mathrm{~nm}^{3}$, and lattice parameters $a=0.2461 \mathrm{~nm}$ and $c=0.6708 \mathrm{~nm}$ for the Rietveld hexagonal GNPs are smaller than the literature values [8,11,34-37].

Table 1. Comparison of hexagonal graphite lattice parameters, cell volume, and density with literature data.

\begin{tabular}{ccccc}
\hline Study & $\boldsymbol{a}(\mathbf{n m})$ & $\boldsymbol{c}(\mathbf{n m})$ & $\mathbf{V ~}\left(\mathbf{n m}^{3}\right)^{*}$ & $\mathbf{Q}\left(\mathbf{g} / \mathbf{c m}^{3}\right)$ \\
\hline This study & 0.24461 & 0.67254 & 0.06996 & 2.28 \\
Lipson et al. [8] & 0.24560 & 0.66960 & 0.07081 & --- \\
Chung et al. [34] & 0.24600 & 0.67100 & 0.07116 & 2.2 \\
Trucano et al. [35] & 0.24620 & 0.67110 & 0.07133 & --- \\
Mohr et al. [36] & 0.24630 & 0.67120 & 0.07142 & --- \\
Li et al. and Hishiyama et al. [11,37] & 0.24612 & 0.67080 & 0.07126 & --- \\
\hline
\end{tabular}

* Cell volume from XRD Rietveld analysis in this study and calculated from a hexagonal cylinder volume in other studies.

The existence of parallel 2D graphene layers with a translational ...ABAB... sequence of tightly bonded $\mathrm{sp}^{2}$ hybridized carbon atoms in hexagonal rings is shown in Figure 6 from the Rietveld crystal lattice of GNP. The soft lubricating nature of the GNPs is provided by the covalently bonded carbon atoms in layers that are bound by weak van der Waals forces, which allows for graphene layer sliding. The distance between adjacent graphene layers in graphite $(0.336 \mathrm{~nm})$ is half of the hexagonal graphite crystallographic spacing $(0.673 \mathrm{~nm})$, which is similar to the calculated (002) peak d-spacing at $2 \theta$ of $26.619^{\circ}$. 


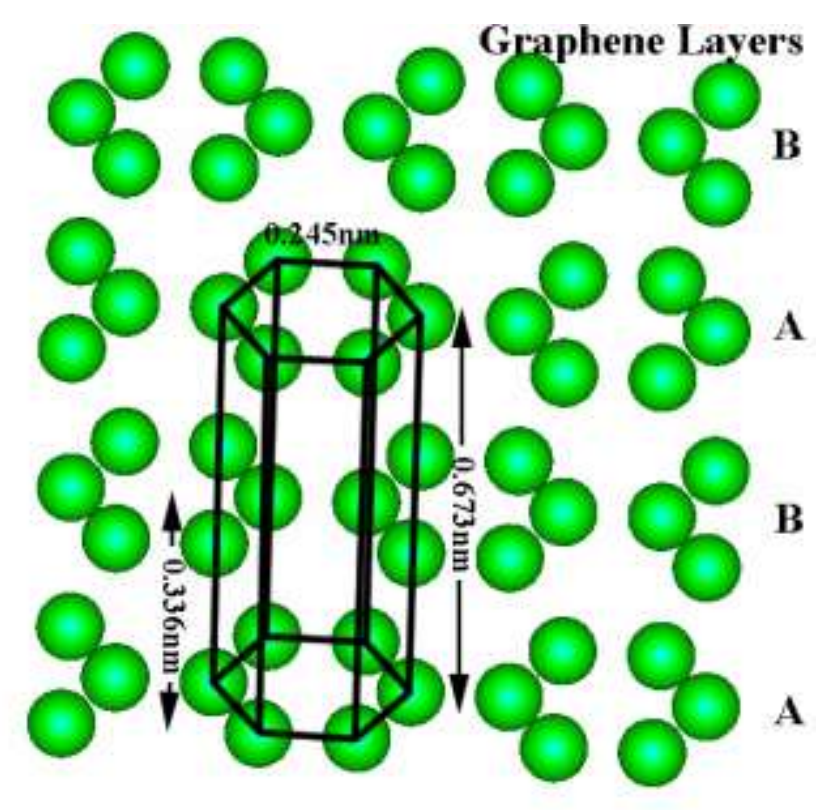

Figure 6. Hexagonal $(2 \mathrm{H})$ GNPs with graphene layers stacked in translational ...ABAB... sequence with room-temperature $0.336 \mathrm{~nm}$ perpendicular interplanar distance.

\section{Conclusions}

Expanded flake graphite was ultrasonicated in isopropyl alcohol to prepare GNPs. XRD, SEM, and TEM were used to study the physicochemical properties of GNP. TEM indicated the formation of a large amount of multilayer and some monolayer GNP by exfoliation. XRD analysis indicated the formation of 43 layers along the c-axis. The GNP crystal structure as determined by XRD and Rietveld refinement indicated nanometer-thick worm-like exfoliated graphite nanoplatelets. The 120-nm-thick graphite units from flaky graphite exfoliation are a promising, low-cost, lightweight alternative to carbon- and metal-based electrically conductive reinforcement applications.

Acknowledgments: Prof. It-Meng Low (Department of Physics and Astronomy, Curtin University) is thanked for manuscript review and feedback. Funding from the Institute for Research and Medical Consultations, Imam Abdulrahman Bin Faisal University, Dammam, Saudi Arabia is gratefully acknowledged.

Conflicts of Interest: The author declares no conflict of interest.

\section{References}

1. Choi, W., Lahiri, I., Seelaboyina, R., Kang, Y.S. Synthesis of graphene and its applications: a review. Crit. Rev. Solid State Mater. Sci. 2010, 35, 52-71.

2. Kim, H., Abdala, A.A., Macosko, C.W. Graphene/polymer nanocomposites. Macromol. 2010, 43, 6515-6530.

3. R. Sengupta, M. Bhattacharya, S. Bandyopadhyay, A.K. Bhowmick. A review on the mechanical and electrical properties of graphite and modified graphite reinforced polymer composites. Prog. Polym. Sci. 2011, 36,638-670.

4. Li, B., Zhong, W.H. Review on polymer/graphite nanoplatelet nanocomposites. J. Mater. Sci. 2011, 46, 5595-5614.

5. Tu, H., Ye, L. Thermal conductive PS/graphite composites. Polym. Adv. Technol. 2009, 20, 21-27.

6. Fukushima, H., Drzal, L., Rook, B., Rich, M. Thermal conductivity of exfoliated graphite nanocomposites. J. Therm. Anal. Calorim. 2006, 85, 235-238.

7. Toyoda, M., Inagaki, M. Heavy oil sorption using exfoliated graphite: New application of exfoliated graphite to protect heavy oil pollution. Carbon. 2000, 38, 199-210.

8. Lipson, H.S., Stokes, A. The structure of graphite. Proc. R. Soc. London, Ser. Math. Phys. Sci. 1942, 181, 101-105.

9. Lipson, H., Stokes, A. A new structure of carbon. Nature. 1942, 149, 328.

10. Bacon, G. The determination of the unit-cell dimensions of non-cubic substances. Acta Crystallogr. 1948, 1, 337.

11. Li, Z., Lu, C., Xia, Z., Zhou, Y., Luo, Z. X-ray diffraction patterns of graphite and turbostratic carbon. Carbon. 2007, 45, 1686-1695. 
12. Salver-Disma, F., Tarascon, J.M., Clinard, C., Rouzaud, J.N. Transmission electron microscopy studies on carbon materials prepared by mechanical milling. Carbon. 1999, 37,1941-1959.

13. Ong, T., Yang, H. Effect of atmosphere on the mechanical milling of natural graphite. Carbon. 2000, 38, 2077-2085.

14. Shen, T.D., Ge, W.Q., Wang, K.Y., Quan, M.X., Wang, J.T., Wei, W.D., Koch, C.C. Structural disorder and phase transformation in graphite produced by ball milling. Nanostruct. Mater. 1996, 7, 393-399.

15. Welham, N., Berbenni, V., Chapman, P. Effect of extended ball milling on graphite. J. Alloys Compd. 2003, 349, 255-263.

16. Zhou, P., Lee, R., Claye, A., Fischer, J.E. Layer disorder in carbon anodes. Carbon. 1998, 36, 1777-1781.

17. Gu, W., Zhang, W., Li, X., Zhu, H., Wei, J., Li, Z., Shu, Q., Wang, C., Wang, K., Shen, W., Kang, F., Wu, D. Graphene sheets from worm-like exfoliated graphite. J. Mater. Chem. 2009, 19, 3367-3369.

18. Delhaes, P. Graphite and precursors: CRC Press; 2000.

19. Chen, G., Weng, W., Wu, D., Wu, C. PMMA/graphite nanosheets composite and its conducting properties. Eur. Polym. J. 2003, 39, 2329-2335.

20. Ganguly, A., George, J.J., Kar, S., Bandyopadhyay, A., Bhowmick, A.K. Rubber nanocomposites based on miscellaneous nanofillers. Curr. Top. Elastomers Res. 2008, 89-99.

21. Chen, G., Weng, W., Wu, D., Wu, C., Lu, J., Wang, P., Chen, X. Preparation and characterization of graphite nanosheets from ultrasonic powdering technique. Carbon. 2004, 42,753-759.

22. Wissler, M. Graphite and carbon powders for electrochemical applications. J. Power Sources. 2006, 156, 142150.

23. Liu, D., Dong, Y., Liu, Y., Ma, N., Sui, G. Cellulose Nanowhisker (CNW)/Graphene Nanoplatelet (GN) Composite Films With Simultaneously Enhanced Thermal, Electrical and Mechanical Properties. Front. Mater. 2019, 6, 235-246.

24. Seehra, M.S., Geddam, U.K., Schwegler-Berry, D., Stefaniak, A.B. Detection and quantification of $2 \mathrm{H}$ and 3R phases in commercial graphene-based materials. Carbon. 2015, 95, 818-823.

25. Low, I.M., Albetran, H.M., Degiorgio, M. Structural Characterization of Commercial Graphite and Graphene Materials. J. Nanotechnol. Nanomater. 2020, 1,23-30.

26. Low, I.M., Albetran, H., Prida, V.M., Vega, V., Manurung, P., Ionescu, M. A comparative study on crystallization behavior, phase stability, and binding energy in pure and $\mathrm{Cr}$-doped $\mathrm{TiO}_{2}$ nanotubes. J. Mater. Res. 2013, 28, 304-312.

27. Yasmin, A., Daniel, I.M. Mechanical and thermal properties of graphite platelet/epoxy composites. Polym. 2004, 45, 8211-8219.

28. Albetran, H., Low, I. Crystallization kinetics study of In-doped and (In-Cr) co-doped $\mathrm{TiO}_{2}$ nanopowders using in-situ high-temperature synchrotron radiation diffraction. Arabian J. Chem. 2020, 13, 3946-3956.

29. Albetran, H., Low, I.M. Effect of indium ion implantation on crystallization kinetics and phase transformation of anodized titania nanotubes using in-situ high-temperature radiation diffraction. J. Mater. Res. 2016, 31, 1588-1595.

30. Albetran, H., O'Connor, B., Prida, V., Low, I. Effect of vanadium ion implantation on the crystallization kinetics and phase transformation of electrospun $\mathrm{TiO}_{2}$ nanofibers. Appl. Phys. A. 2015, 120, 623-634.

31. Albetran, H., Low, I.M. Crystallization kinetics and phase transformations in aluminum ion-implanted electrospun $\mathrm{TiO}_{2}$ nanofibers. Appl. Phys. A. 2016, 122, 1044-1053.

32. Albetran, H., Vega, V., Prida, V.M., Low, I.M. Dynamic Diffraction Studies on the Crystallization, Phase Transformation, and Activation Energies in Anodized Titania Nanotubes. Nanomaterials. 2018, 8, 122-131.

33. Albetran, H., Haroosh, H., Dong, Y., Prida, V., O'Connor, B., Low, I.M. Phase transformations and crystallization kinetics in electrospun $\mathrm{TiO}_{2}$ nanofibers in air and argon atmospheres. Appl. Phys. A. 2014, 116, 161-169.

34. Chung, D. Review graphite. J. Mater. Sci. 2002, 37, 1475-1489.

35. Trucano, P., Chen, R. Structure of graphite by neutron diffraction. Nature. 1975, 258, 136-137.

36. Mohr, M., Maultzsch, J., Dobardzic, E., Reich, S., Milosevic, I., Damnjanovic, M., Bosak, A., Krisch, M., Thomsen, C. Phonon dispersion of graphite by inelastic X-ray scattering. Phys. Rev. B. 2007, 76, 035439035436.

37. Hishiyama, Y., Inagaki, M. Lattice parameter changes in graphite with boron doping. Carbon (New York, NY). 2001, 39, 150-152. 